\title{
Análise da confiabilidade do método Figura Oito e da volumetria para mensuração do edema de tornozelo
}

Filipe Abdalla dos Reis¹, Eduardo Alves Ribeiro', Paulo de Tarso Camillo de Carvalho ${ }^{1,2}$, Ana Carulina Guimarães Belchior ${ }^{1}$, Juliano Coelho Arakaki² e Rodrigo Antunes de Vasconcelos ${ }^{2}$

\section{RESUMO}

A presença de lesões no tornozelo torna necessária a mensuração do edema como fator diagnóstico e evolutivo. O presente trabalho teve como objetivo analisar a confiabilidade do método Figura Oito e da volumetria para a mensuração do volume do tornozelo, intrateste e interteste. Vinte voluntários, sexo masculino, assintomáticos, idade entre 15 e 30 anos $(\bar{X}=21,8)$, foram avaliados aleatoriamente por três examinadores; cada um realizou três mensurações utilizando o método Figura Oito e três, a volumetria. A ordem dos examinadores e da técnica de mensuração foi aleatória. Observou-se excelente confiabilidade nas duas técnicas (ICC $=0,99$ ). O coeficiente de correlação momento-produto de Pearson demonstrou similaridade entre os examinadores; o primeiro apresentou $r$ $=0,91$, o segundo, $r=0,95$ e o terceiro, $r=0,96$. Concluiu-se que os dois métodos são confiáveis, apesar de a maioria dos autores recomendar o método Figura Oito pela sua praticidade, rapidez e baixo custo.

\section{RESUMEN}

\section{Análisis de la confiabilidad del método Figura Ocho y de la volumetria para medida del edema de tobillo}

La presencia de lesiones del tobillo hace necesario el medir la magnitud del edema como factor diagnóstico y evolutivo. El presente trabajo tiene como objetivo analisar la confiabilidad del metodo Figura Ocho y de la volumetría para la mensura del volumen del tobillo intratest e interteste. Veinte voluntarios, de sexo masculino, asintomáticos, de edades entre 15 a 30 años $(\bar{X}=21.8)$, fueron avaluados aleatoriamente por tres examinadores, donde cada uno realizó tres medidas utilizando el método Figura Ocho y tres utilizando la volumetria. La orden de los examinadores y de la técnica de mensura fué aleatoria. Se ha observado una excelente confiabilidad en las dos técnicas (ICC $=0.99$ ). El coeficiente de correlación momento-produto de Pearson demonstró similaridad entre los examinadores, siendo que el primero presentó $r=0.91$, el segundo $r=0.95$ y el tercero $r=0.96$. Se concluye que los dos métodos son confiables, a pesar de la mayoría de los autores han recomendado el método Figura Ocho por su practicidade, rapidez e bajo costo.

1. Curso de Fisioterapia da Universidade para o Desenvolvimento do Estado e da Região do Pantanal - Uniderp - Campo Grande/MS.

2. Departamento de Biomecânica, Medicina e Reabilitação do Aparelho Locomotor - Faculdade de Medicina de Ribeirão Preto da Universidade de São Paulo - FMRP-USP.

Recebido em 24/9/04. 2a versão recebida em 17/11/04. Aceito em 17/11/04.

Endereço para correspondência: Rua Goiás, 1.709, Vila Célia - Campo Grande, MS, Brasil. Tel.: (67) 3029-0642, e-mail: fi_abdalla@pop.com.br e Universidade para o Desenvolvimento do Estado e da Região do Pantanal Uniderp, Av. Fernando Corrêa da Costa, 4.000 - Campo Grande, MS, Brasil. Tel.: (67) 348-8162.
Palavras-chave: Tornozelo. Edema. Mensuração.

Palabras-clave: Tobillo. Edema. Mensuración.

\section{INTRODUÇÃO}

Lesões no tornozelo decorrentes de traumas são freqüentes durante a prática esportiva, levando o atleta ao afastamento das competições. Grande parte dessas lesões resulta em processo inflamatório agudo, no qual o edema é um dos sinais mais relevantes $^{(1-4)}$.

O edema indica reação inflamatória tecidual, alteração na dinâmica normal dos capilares e funcionamento inadequado ou comprometido do mecanismo de bombeamento venoso e do sistema linfático(5,6).

Denota-se excesso de líquido no interstício ou em cavidades serosas; sendo um processo desencadeado pela histamina, pela bradicinina, pelos leucotrienos e por outras classes de mediadores químicos, pode corresponder a um exsudato ou a transudato. $\mathrm{O}$ exsudato é o líquido inflamatório extravascular com concentração protéica elevada e grande quantidade de restos celulares; em contraste, o transudato é um líquido com teor protéico baixo composto em sua maior parte de albumina ${ }^{(7,8)}$.

Petersen et al.(9) classificaram o edema do tornozelo, por método visual, em: leve, moderado e grave. O edema leve ocorre quando todos os marcos ósseos (maléolos, osso navicular e base do quinto metatarso) estão bem visíveis; o moderado, quando os marcos ósseos são menos visíveis; e o grave, quando os marcos ósseos têm difícil localização visual.

Gabriel et al.(10) relatam que, caso o edema não seja corrigido, acarretará estado de incapacidade funcional pela limitação da elasticidade muscular, diminuição dos arcos articulares, encurtamento de aponeuroses e, em certos casos, necrose tecidual.

Com associação de métodos de mensuração do edema, podese avaliar a gravidade da lesão e efetividade do tratamento; vários métodos são utilizados para mensurar o volume dos membros, sendo eles: medida de circunferência, volumetria, impedância bioeletrônica e modelo computacional| $(9,11,12)$.

A volumetria e o método Figura Oito são os processos avaliativos padronizados mais utilizados para mensuração do edema do tornozelo com finalidade de quantificar a eficácia do tratamento ${ }^{15,11-}$ 15).

Desde o tempo de Arquimedes, o deslocamento da água é utilizado para mensurar a massa corporal e relatos na literatura indicam que foi útil para diferenciar o ouro de outros metais para a criação da coroa do rei Hiero(5,12).

A volumetria é uma importante técnica para mensurar o edema articular em superfícies irregulares como o pé e tornozelo. A mensuração é realizada pelo volúmetro, um recipiente translúcido especialmente projetado, contendo água em seu interior e com uma calha para extravasamento desse líquido(12,14-17).

O método Figura Oito, desenvolvido por Esterson(18), consiste da perimetria, com fita métrica, das áreas de maior concentração de edema no tornozelo, ou seja, a região dos ligamentos talofibular anterior, calcaneofibular e tibiofibular anterior ${ }^{(11)}$. 
Mawdsley et al.(13) determinaram que o método Figura Oito tem confiabilidade baseada no coeficiente de correlação intraclasse, porém sua validade em relação à volumetria ainda não foi estabelecida.

Assim, o presente trabalho teve como objetivo analisar a confiabilidade e aplicabilidade do método Figura Oito e da volumetria para a mensuração do volume do tornozelo na prática clínica, intrateste e interteste.

\section{CASUÍSTICA E MÉTODOS}

\section{Amostra}

Foram recrutados, por convite verbal, 20 voluntários, sexo masculino, idade entre 15 e 30 anos ( $\bar{X}=21,8)$, sem antecedentes traumáticos. Foram excluídos da seleção da amostra os voluntários que apresentaram distúrbios traumato-ortopédicos prévios (fraturas, tendinopatias), procedimento cirúrgico prévio e outras patologias sistêmicas.

Antes do início do experimento, todos os voluntários foram esclarecidos sobre os procedimentos da pesquisa, com assinatura de Termo de Consentimento Livre e Informado segundo as Diretrizes e Normas Regulamentadoras de Pesquisa envolvendo Seres Humanos constantes da Resolução do Conselho Nacional de Saúde № 196/96.

\section{Procedimentos}

\section{Mensuração volumétrica}

Utilizou-se volúmetro manufaturado em material translúcido (vidro), segundo o padrão da marca Baseling Volumetric Edema Set, Idyllwild, CA, com dimensões: $33,5 \mathrm{~cm}$ de comprimento, $13 \mathrm{~cm}$ de largura e $24 \mathrm{~cm}$ de altura (fig. 1).

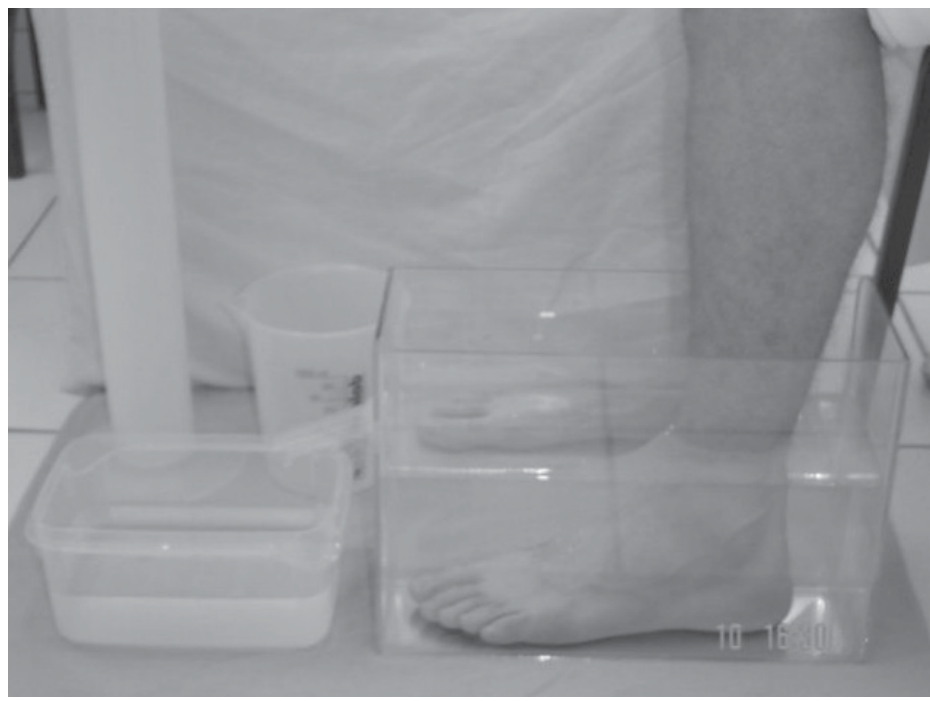

Fig. 1 - Mensuração volumétrica: $\boldsymbol{A})$ volúmetro, B) recipiente, C) proveta, D) Becker.

Os voluntários em trajes apropriados, expondo pernas e tornozelos, foram posicionados em cadeira mantendo flexão de joelho esquerdo, selecionado aleatoriamente, em aproximadamente $90^{\circ}$. Antes da imersão, uma camada de água foi aplicada manualmente ao membro inferior esquerdo (MIE) para minimizar a quantidade de ar contida sob os pêlos ${ }^{(15)}$

A seguir, os voluntários foram instruídos a abaixar suavemente o MIE no volúmetro, contendo cinco litros de água à temperatura ambiente, até que o pé descansasse confortavelmente no fundo. O volume de água deslocado foi descarregado por uma calha em um Becker e transferida para uma proveta de $1.000 \mathrm{~mL}$ (graduação de $10 \mathrm{~mL})$.
Aleatoriamente, três examinadores clínicos ( $A$, B e C) foram selecionados para a coleta individual da volumetria. Cada examinador realizou a mensuração três vezes em cada voluntário, registrando os valores em formulário padronizado.

\section{Método Figura Oito}

Os examinadores instruíram os voluntários, também aleatoriamente, a permanecerem sentados com o joelho em extensão completa e o tornozelo em posição neutra. Realizou-se a mensuração, o zero da fita métrica mantido sobre o ponto médio entre a projeção articular do tendão do tibial anterior e o maléolo lateral. Direcionou-se a fita para o centro do arco longitudinal medial do pé, sobre o osso navicular, tangenciando a região plantar em direção ao maléolo lateral, tendão calcâneo e maléolo medial até encontrar o ponto zero da fita (fig. 2).

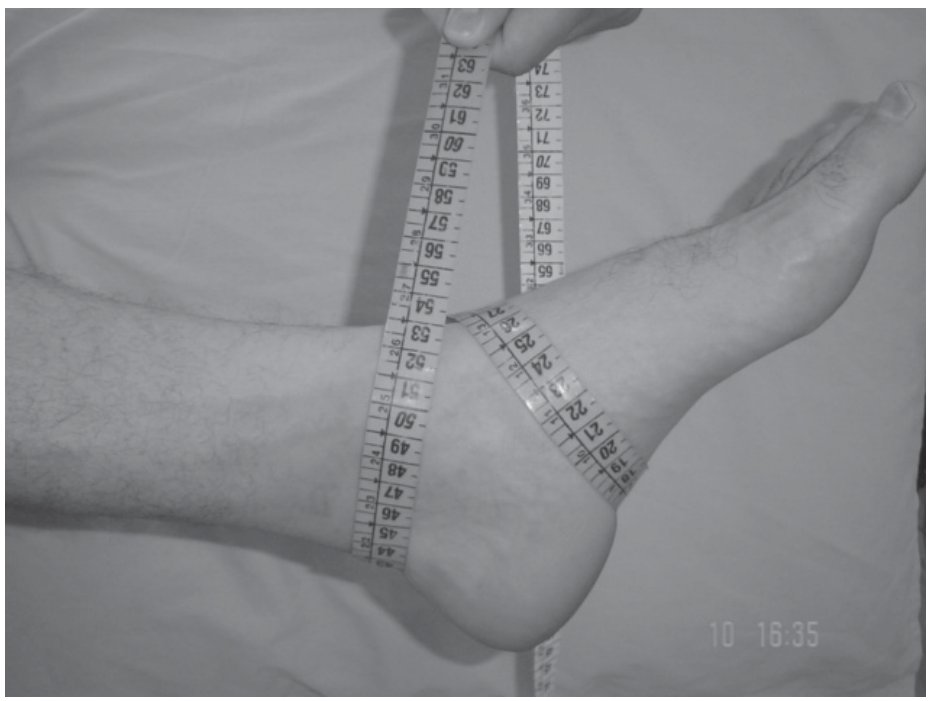

Fig. 2 - Método Figura Oito: $\boldsymbol{A}$ ) fita métrica, B) arco longitudinal medial do pé, $\boldsymbol{C}$ tendão calcaneano, $\boldsymbol{D}$ ) maléolo medial.

Cada examinador realizou a mensuração três vezes em cada voluntário, registrando os valores em formulário padronizado.

\section{Análise estatística}

Aplicou-se a análise da variância (ANOVA) com finalidade de comparar as médias obtidas pelos examinadores, individualmente e entre si.

Calculou-se o coeficiente de correlação momento-produto de Pearson, para medir o grau de relacionamento linear entre as medidas coletadas da volumetria e do método Figura Oito, para cada examinador.

Para a avaliação da confiabilidade dos métodos, aplicou-se o teste $F$ determinando a diferença entre as variâncias, ou seja, em qual método houve menor variação na coleta de dados pelos examinadores. Outro teste utilizado para verificar a confiabilidade dos métodos de coleta foi o cálculo do coeficiente de correlação intraclasse (ICC), no qual valores mais próximos de 1 indicam excelente confiabilidade do teste.

\section{RESULTADOS}

Na análise da variância (ANOVA) não houve diferença significativa para a volumetria $(p=0,87)$ e para o método Figura Oito $(p=$ $0,12)$.

Em relação ao coeficiente de correlação momento-produto de Pearson, os examinadores obtiveram similaridade de resultados, estando a menor variação na coleta do examinador C (tabela 1).

O resultado do teste $F$ apresentou, em $90 \%$ dos indivíduos, variabilidade maior na volumetria $(p<0,05)$ e, em 10\% dos indiví- 
duos, houve a mesma variabilidade. Em nenhum indivíduo a variabilidade da volumetria foi menor do que no método Figura Oito.

O conjunto de dados, após tratamento estatístico, demonstrou que há menor variabilidade nos valores coletados pelos examinadores no método Figura Oito (gráfico 1) e o resultado do ICC de 0,99 para os dois métodos de mensuração confirma a confiabilidade de ambos.

\section{TABELA 1}

Coeficiente de correlação momento-produto de

Pearson ( $r$ ) entre as médias dos três examinadores

\begin{tabular}{cc}
\hline Examinador & $\mathbf{r}^{*}$ \\
A & 0,91 \\
B & 0,95 \\
C & 0,96 \\
\hline
\end{tabular}

* $p<0,01$

A

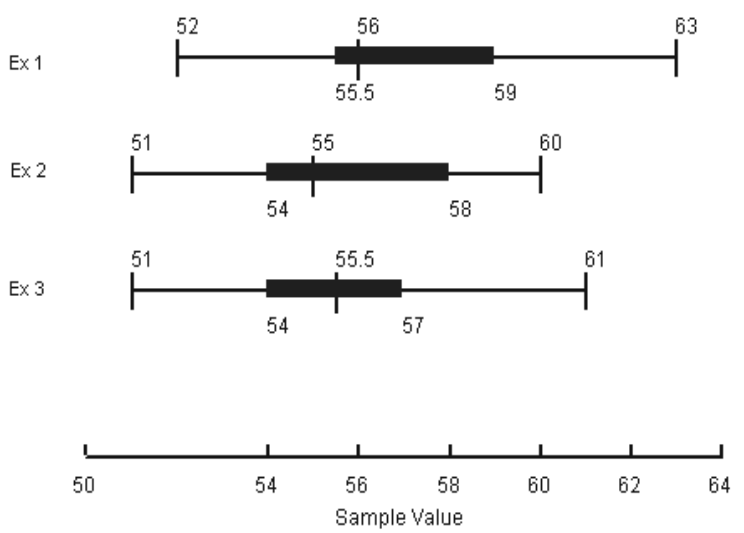

B
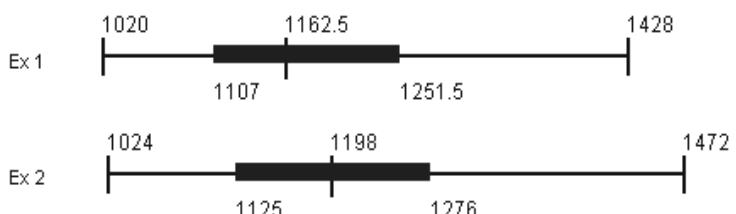

1125

1276

Ex 3
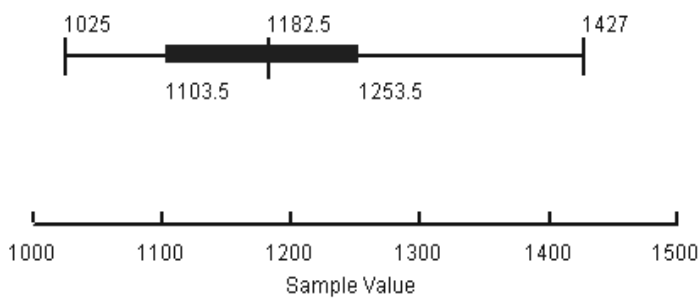

Gráfico 1 - Média dos valores coletados pelos examinadores: $\boldsymbol{A}$ ) método Figura Oito; B) volumetria.

\section{DISCUSSÃo}

A validação dos resultados baseou-se na homogeneidade da amostra, composta de adultos jovens do sexo masculino, com idade média de 21,8 anos, valores que confirmam os dados encontrados na literatura ${ }^{(9,13,19-31)}$.

Os resultados indicam que ambos os métodos são fidedignos na mensuração do edema de tornozelo, presumindo-se que esses resultados devem-se à padronização das mensurações.
Acredita-se que a volumetria apresentou maior variabilidade no teste $F$ devido à instabilidade do meio líquido, visto que a maioria dos voluntários avaliados não conseguia permanecer com o MIE imóvel durante a realização da mensuração.

Pressupõe-se que a similaridade de resultados foi decorrente do treinamento prévio dos examinadores e do aperfeiçoamento técnico individual.

Mawdsley et al.(13) propuseram que o método Figura Oito poderia ser confiável, obtendo ICC de 0,99 ao mensurar 15 indivíduos com edema secundário a uma entorse crônica de tornozelo. TatroAdams et al.(11) obtiveram resultado igual ao mensurar 50 indivíduos com entorse de tornozelo, obtendo ICC de 0,99.

Wilson et al.(15) observaram ICC de 0,95 ao avaliar 34 indivíduos atletas com entorse de tornozelo.

Em concordância com Esterson ${ }^{(18)}$ e Tatro-Adams et al.(11) o método Figura Oito é uma técnica de fácil execução, rapidez, baixo custo e confiabilidade e, quando comparado com a volumetria, é também mais higiênica.

Entretanto, presume-se que o Figura Oito não deve ser escolhido como método de mensuração quando o edema atingir toda a extremidade inferior, como porção distal da perna, tornozelo e pé, sendo nestes casos recomendada a volumetria.

Outros trabalhos, comparando diferentes métodos de mensuração, devem ser implementados para melhor avaliação dos resultados em função da escassez de trabalhos na literatura pesquisada (Medline, Lilacs).

\section{CONCLUSÃo}

Conclui-se que o Figura Oito e a volumetria são métodos de alta confiabilidade e de fácil aplicabilidade clínica na mensuração do volume da articulação do tornozelo.

Todos os autores declararam não haver qualquer potencial conflito de interesses referente a este artigo.

\section{REFERÊNCIAS}

1. Siegler S, Liu W, Sennett B, Nobilini RJ, Dunbar D. The three-dimensional passive support characteristics of ankle braces. J Orthop Sports Phys Ther 1997; 26:299-309.

2. Robbins S, Waked E. Fatores associados a lesões no tornozelo-Medidas preventivas. Sprint Magazine 1999;7:4-13.

3. Uh BS, Beynnon BD, Helie BV, Alosa DM, Renstrom PA. The benefit of a singleleg strength-training program for the muscles around the untrained ankle. Am J Sports Med 2000;28:568-73.

4. Dijk CNV. Management of the sprained ankle. Br J Sports Med 2002;36:83-4.

5. Devore GL, Hamilton GF. Volume measuring of the severely injured hand. Am J Orthop Trauma 1968;12:16-8.

6. Stern EB. Volumetric comparison of seated and standing test postures. Am J Occup Ther 1991;45:801-5.

7. Kitchen S, Bazin S. Eletroterapia de Clayton. São Paulo: Manole, 1998.

8. Low J, Reed A. Eletroterapia explicada: princípios e prática. 3a ed. Barueri: Manole, 2001

9. Petersen EJ, Irish SM, Lyons CL, Miklaski SF, Bryan JM, Henderson NE, et al. Reliability of water volumetry and the figure of eight method on subjects with ankle joint swelling. J Orthop Sports Phys Ther 1999;29:609-15.

10. Gabriel MRS, Petit JD, Carril MLS. Fisioterapia em traumatologia, ortopedia e reumatologia. Rio de Janeiro: Revinter, 2001.

11. Tatro-Adams D, McGann SF, Carbone W. Reliability of the figure-of-eight method of ankle measurement. J Orthop Sports Phys Ther 1995;22:161-3.

12. Moholkar K, Fenelon G. Diurnal variations in volume of the foot and ankle. J Foot Ankle Surg 2001;40:302-4.

13. Mawdsley RH, Hoy DK, Erwin PM. Criterion-related validity of the figure-of-eight method of measuring ankle edema. J Orthop Sports Phys Ther 2000;30:149-53.

14. Cloughley WB, Mawdsley RH. Effect of running on volume of the foot and ankle. J Orthop Sports Phys Ther 1995;22:151-4.

15. Wilson RW, Gieck JH, Gansneder BM, Perrin DH, Saliba EN, McCue FC 3rd. Reliability and responsiveness of disablement measures following acute ankle sprains among athletes. J Orthop Sports Phys Ther 1998;27:348-55. 
16. O'Sulivan S, Scmitz TJ. Fisioterapia: avaliação e tratamento. 2a ed. São Paulo: Manole, 1993.

17. Wester JU, Jespersen SM, Nielsen KD, Neumann L. Wobble board training after partial sprains of the lateral ligaments of the ankle: a prospective randomized study. J Orthop Sports Phys Ther 1996;23:332-6.

18. Esterson PS. Measurement of ankle joint swelling using a figure-of-eight. J Orthop Sports Phys Ther 1979;1:51-2.

19. Goldie IF, Gunterberg B, Jacobson C. Foot volumetry as an objective test of the effect of antiphlogistic drugs in ankle sprains. A preliminary study. Rheumatol Rehabil 1974;13:204-7.

20. Wilkerson GB, Pinerola JJ, Caturano RW. Invertor vs. evertor peak torque and power deficiencies associated with lateral ankle ligament injury. J Orthop Sports Phys Ther 1997;26:78-86.

21. Matsusaka N, Yokoyama S, Tsurusaki T, Inokuchi S, Okita M. Effect of ankle disk training combined with tactile stimulation to the leg and foot on functional instability of the ankle. Am J Sports Med 2001;29:25-30.

22. Kovaleski JE, Gurchiek LR, Heitman RJ, Hollis JM, Pearsall AW. Instrumented measurement of anteroposterior and inversion-eversion laxity of the normal ankle joint complex. Foot Ankle Int 1999;20:808-15.

23. Verbrugge JD. The effects of semirigid air-stirrup bracing vs. adhesive ankle taping on motor performance. J Orthop Sports Phys Ther 1996;23:320-5.
24. Guskiewicz KM, Perrin DH. Effect of orthotics on postural sway following inversion ankle sprain. J Orthop Sports Phys Ther 1996;23:326-31.

25. Forkin DM, Koczur C, Battle R, Newton RA. Evaluation of kinesthetic deficits indicative of balance control in gymnasts with unilateral chronic ankle sprains. J Orthop Sports Phys Ther 1996;23:245-50

26. Ebig M, Lephart SM, Burdett RG, Miller MC, Pincivero DM. The effect of sudden inversion stress on EMG activity of the peroneal and tibialis anterior muscles in the chronically unstable ankle. J Orthop Sports Phys Ther 1997;26:73-7.

27. Heidt RS, Sweeterman LM, Carlonas RL, Traub JA, Tekulve FX. Avoidance of soccer injuries with preseason conditioning. Am J Sports Med 2000;28:659-62.

28. Refshauge KM, Kilbreath SL, Raymond J. The effect of recurrent ankle inversion sprain and taping on proprioception at the ankle. Med Sci Sports Exerc 2000;32: 10-5.

29. Eiff MP, Smith AT, Smith GE. Early mobilization versus immobilization in the treatment of lateral ankle sprains. Am J Sports Med 1994;22:83-8.

30. Thacker SB, Stroup DF, Branche CM, Gilchrist J, Goodman J, Weitman EA. The prevention of ankle sprains in sports: a systematic review of the literature. Am J Sports Med 1999;27:753-60.

31. Hashimoto T, Suguru L. A kinematic study of ankle joint instability due to rupture of the lateral ligaments. Foot Ankle Int 1997;18:729-34. 